\title{
Panorama actual de la fibrosis pulmonar idiopática en México
}

\author{
José O. Barreto-Rodríguez, Mayra E. Mejía, Ivette Buendía-Roldán $\bowtie$
}

Instituto Nacional de Enfermedades Respiratorias Ismael Cosío Villegas, Ciudad de México.

Trabajo recibido: 10-VIII-2015; aceptado: 14-VIII-2015

\begin{abstract}
RESUMEN. La fibrosis pulmonar idiopática (FPI) es una patología caracterizada por fibrosis pulmonar progresiva e irreversible de etiología desconocida, su incidencia y prevalencia en el mundo es muy variable. En México no contamos con datos epidemiológicos representativos. Se aplicó una encuesta a 419 neumólogos, de los cuales respondieron 168 neumólogos de todo el país. En promedio cada neumólogo de México diagnostica 8 pacientes con FPI por año. Los centros de referencia que reconocen los neumólogos son: $27 \%$ al INER, $6 \%$ CMN IMSS, $2 \%$ HGM, $2 \%$ CEPREP y $63 \%$ negó tener centro de referencia. Para el abordaje diagnóstico, $89 \%$ dispone de TAC-AR y $11 \%$ no dispone de ella; $37 \%$ tiene apoyo de un radiólogo experto; $31 \%$ de un patólogo experto, y $63 \%$ no dispone de estos especialistas. Con respecto al tratamiento de inicio $85 \%$ prescribe prednisona, $46 \%$ n-acetilcisteína, $39 \%$ azatioprina, $12 \%$ colchicina y $8 \%$ pirfenidona. Con los resultados obtenidos en esta encuesta podemos concluir que México no sólo necesita formar neumólogos expertos en enfermedades intersticiales sino que, además, es fundamental incrementar la cantidad de radiólogos y patólogos expertos en el tema, así como formar una red adecuada de centros de referencia nacional para obtener datos epidemiológicos confiables.
\end{abstract}

Palabras clave: Fibrosis pulmonar idiopática, México, encuesta.

ABSTRACT. IPF is a disease characterized by progressive and irreversible pulmonary fibrosis of unknown etiology, incidence and prevalence in the world is highly variable; in Mexico we have no representative epidemiological data. An inquiry was conducted of 419 pulmonologists, of which 168 responded pulmonologists around the country. On average each pulmonologist diagnosed 8 patients with IPF annually. Reference centers that recognize are INER 27\%, 6\% CMN IMSS, HGM 2\%, 2\% CEPREP, and 63\% denied having reference center. For the diagnostic approach $89 \%$ has HRCT and $11 \%$ do not have it, $37 \%$ is support of an expert radiologist, $31 \%$ of an expert pathologist and $63 \%$ do not have these specialists. Regarding the initial treatment prescribed prednisone $85 \%, 46 \%$ n-acetilcisteine, $39 \%$ azatioprine. Colchicine $12 \%$ and $8 \%$ pirfenidone. With the results of this study we can conclude that Mexico needs not only train experts pulmonologists in interstitial diseases, it is also essential to increase the number of radiologists and expert pathologists in the subject and form an adequate network of national reference for obtain reliable epidemiological data.

Key words: Idiopathic pulmonary fibrosis, Mexico, inquiry.

\section{INTRODUCCIÓN}

La fibrosis pulmonar idiopática (FPI) se define como una neumonía intersticial usual de causa desconocida, que cursa con fibrosis pulmonar crónica y progresiva, de predominio en el adulto mayor, y con una sobrevida media de 3-5 años posterior al diagnóstico. ${ }^{1,2}$ Su incidencia en el mundo oscila entre 0.6 a 17.4 casos $/ 100,000 /$ año, mientras que la prevalencia varía de 0.7 a 63 casos por cada 100,000 habitantes. ${ }^{3}$

El diagnóstico se realiza al excluir causas probables de fibrosis pulmonar aunado a un patrón de neumonía intersticial usual por tomografía de alta resolución (TACAR) y/o histopatología. ${ }^{1}$ Por tal motivo y de acuerdo con consenso de 2011 ATS/ERS/JRS/ALAT de fibrosis pulmonar idiopática se ha mostrado que el diagnóstico debe ser multidisciplinario e involucrar al radiólogo, patólogo y neumólogo.

Por otro lado y con respecto al abordaje terapéutico, éste sigue siendo un reto, ya que al igual que para el diagnóstico se requiere un manejo sistemático que incluya tratamiento de comorbilidades, oxigenoterapia, rehabilitación pulmonar y en el último año, el tratamiento farmacológico con moléculas antifibróticas como nintedanib $^{4}$ y/o pirfenidona. ${ }^{5}$

Debido a todas estas dificultades diagnóstico-terapéuticas y a las diferencias reportadas en la incidencia y prevalencia de la enfermedad, se consideró interesante 
conocer la frecuencia con la que los neumólogos de México diagnostican esta enfermedad, así como identificar la problemática que se tiene tanto para el diagnóstico como para el tratamiento, lo que nos permitirá conocer el panorama actual con respecto a FPI en nuestro país y tomar las medidas necesarias para mejorar la situación.

\section{MATERIAL Y MÉTODOS}

Con el apoyo del grupo experto en enfermedades intersticiales del pulmón del Instituto Nacional de Enfermedades Respiratorias Ismael Cosío Villegas (INER) de la Ciudad de México, se diseñó una encuesta de 22 reactivos sobre FPI. En el mes de octubre de 2013 se aplicó vía on-line a 419 neumólogos de México; sin embargo, debido a la baja respuesta (11/419) se decidió su impresión y nuevamente fue realizada por escrito en mayo de 2014 en el Congreso Nacional de Neumología y Cirugía de Tórax donde respondieron 157 neumólogos diferentes a los primeros, obteniendo una respuesta final de 168 neumólogos de todo el país. Los resultados fueron registrados y analizados con el programa Excel Versión 14.4.7.

\section{RESULTADOS}

Se encuestaron 419 neumólogos de México, de los cuales 11 respondieron vía on-line en 2013 y 157 más en el Congreso Nacional de Neumología y Cirugía de Tórax realizado en la Ciudad de Querétaro en mayo de 2014, obteniendo una respuesta final de 168 neumólogos. El $32 \%$ tenía entre 0-10 años ejerciendo la neumología, $23 \%$ entre $11-20$ años, $24 \%$ entre $21-30$ años y $21 \%$ más de 30 años (figura 1).

En promedio cada neumólogo de México diagnostica 8 pacientes con FPI por año $( \pm 8)$; aunque, al analizar el resultado entre los diferentes estados del país pudieron separarse de la siguiente manera (figura 2, [Nota: Los colores hacen referencia a la versión en línea]): en diagonales (verde) los estados donde los neumólogos diagnostican por año entre 1-5 pacientes con FPI; en puntos (azul) entre 6-10 pacientes/año; en difuminado (amarillo) entre 11-15 pacientes/año; y en barras (rojo)

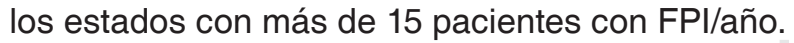

En cuanto a los centros de referencia que reconocen los neumólogos para pacientes con enfermedades intersticiales pulmonares, $63 \%$ dijo no tener centro de referencia, $27 \%$ refiere al INER, $6 \%$ mencionó algún Centro Médico Nacional del Instituto Mexicano del Seguro Social (CMN, IMSS), 2\% reportó al Hospital General de México (HGM) y 2\% al Centro de Prevención y Rehabilitación de Enfermedades Pulmonares Crónicas (CEPREP) en la Ciudad de Monterrey (figuras 3 y 4).

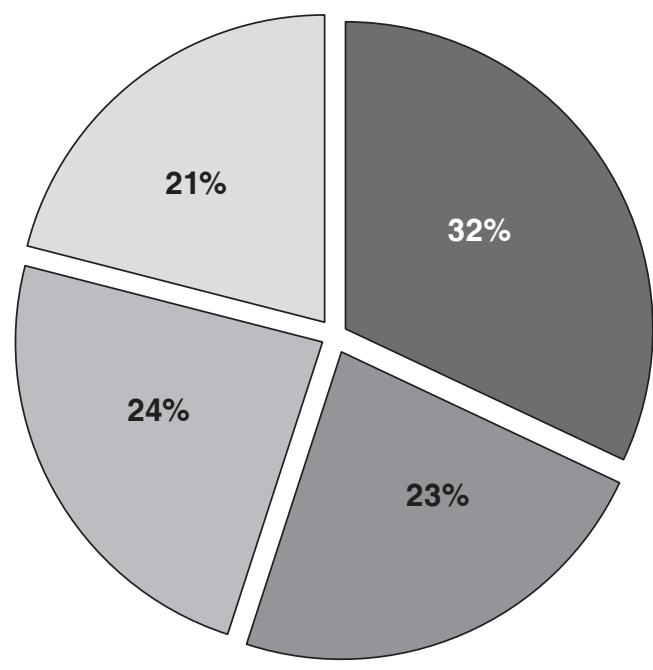

0-10 años

11-20 años

21-30 años

$>30$ años

Fuente: Primera encuesta nacional de fibrosis pulmonar idiopática.

Figura 1. Años de práctica neumológica en los médicos encuestados.

Con respecto a la disponibilidad de tomografía de alta resolución (TAC-AR) y el apoyo multidisciplinario del patólogo y/o radiólogo expertos para realizar el diagnóstico de la enfermedad respondieron lo siguiente: $89 \%$ dispone de TAC-AR y sólo $11 \%$ no dispone de ella. $63 \%$ no dispone de radiólogo ni de patólogo experto en enfermedades intersticiales, 37\% dispone de radiólogo y $31 \%$ de algún patólogo experto, con una distribución de los mismos de predominio en el centro del país, como se observa en la figura 4.

Para el abordaje diagnóstico respondieron que además de la TAC-AR solicitan a sus pacientes anticuerpos para descartar colagenopatías y antígeno aviario para descartar neumonitis por hipersensibilidad, siendo los resultados de la siguiente manera: $33 \%$ solicita factor reumatoide (FR), 54\% anticuerpos antinucleares (ANAs), 12\% anticuerpos antiDNA de doble cadena, $12 \%$ anti-Ro/Anti-La, $8 \%$ anti-Scl $70,9 \%$ anticuerpos antipéptido cíclico citrulinado, $3 \%$ antígeno aviario y $31 \%$ no solicitan ningún anticuerpo (figura 5).

Por otro lado, las comorbilidades con más frecuencia vistas por los neumólogos en sus pacientes con FPI están: $52 \%$ diabetes mellitus, $40 \%$ hipertensión arterial sistémica, 35\% enfermedad pulmonar obstructiva crónica, $27 \%$ hipertensión pulmonar, 5\% síndrome de apnea obstructiva del sueño y $3 \%$ enfermedad por reflujo gastroesofágico (figura 6). 


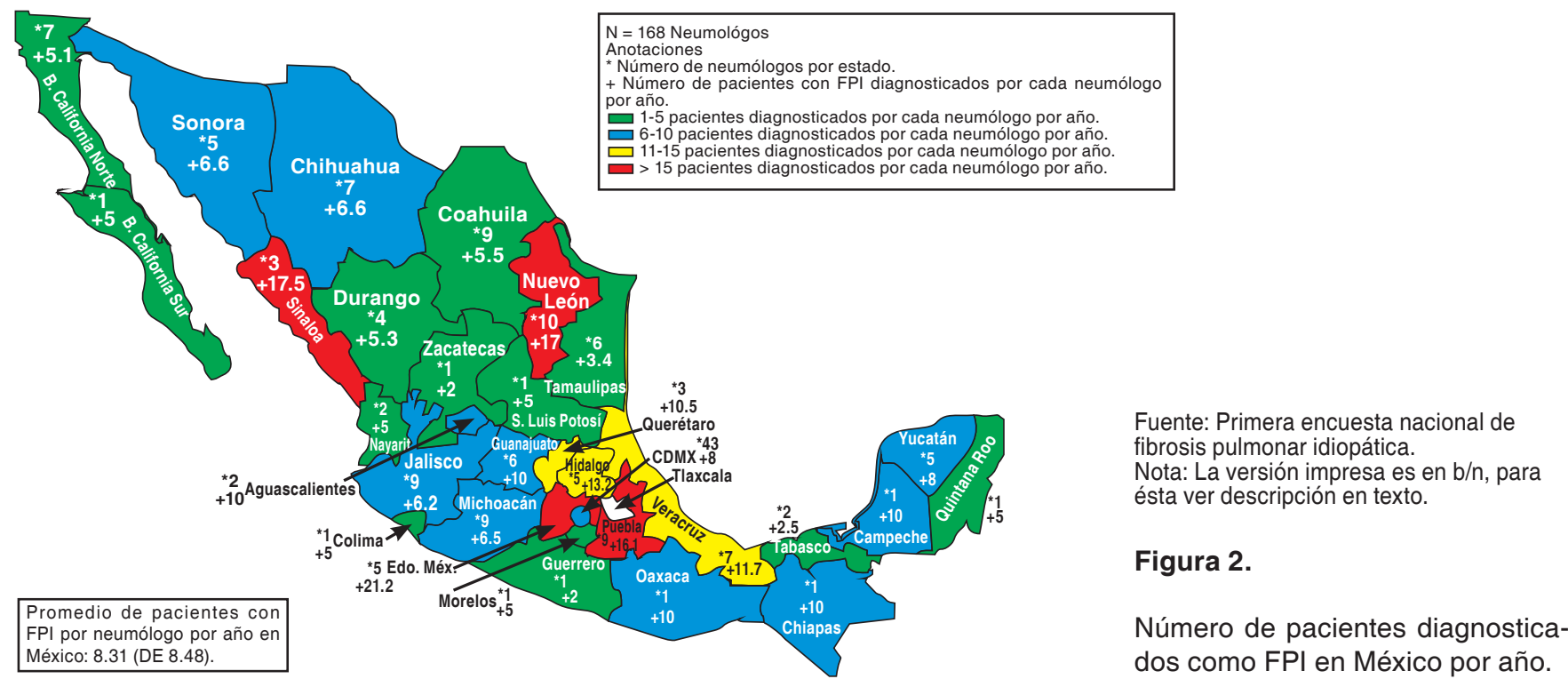

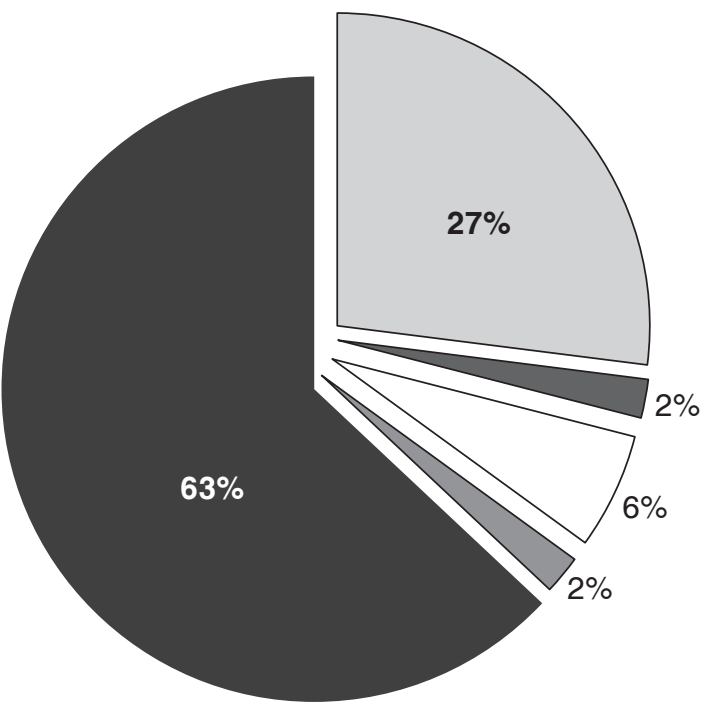

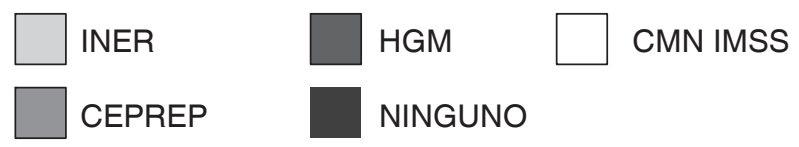

Fuente: Primera encuesta nacional de fibrosis pulmonar idiopática.

Figura 3. Centros de referencia para enfermedades intersticiales del pulmón.

Finalmente, con respecto al tratamiento de inicio para sus pacientes con $\mathrm{FPI}, 85 \%$ refiere iniciar con prednisona, $46 \%$ con n-acetilcisteína (NAC), 39\% con azatioprina, $12 \%$ con colchicina, $8 \%$ con pirfenidona y $0.5 \%$ con factor de transferencia. Las combinaciones más frecuentemente utilizadas como terapia de inicio fueron las siguientes: Prednisona/azatioprina/NAC $34 \%$, prednisona/azatioprina $3 \%$ y prednisona/NAC $6 \%$ (figuras 7 y 8 ).

\section{DISCUSIÓN}

La FPI es una enfermedad en vías de investigación con una alta dificultad diagnóstica/terapéutica, por lo que los datos epidemiológicos a nivel mundial son muy variados y en México inexistentes; sin embargo, debido a su alta tasa de mortalidad (61.2 por cada millón en hombres) ${ }^{6}$ representa una gran problemática, tanto para el paciente como para su médico tratante.

Con base en lo anterior, es momento de que en nuestro país se establezcan las medidas necesarias que permitan un mejor abordaje diagnóstico/terapéutico en enfermedades intersticiales del pulmón y, con ello, el conocimiento del impacto epidemiológico que representan, para lo que proponemos el inicio de un registro nacional de pacientes con FPI. No obstante, todo gran trabajo requiere un análisis previo que nos permita identificar los puntos clave que se deben considerar al momento de decidir mejorar el panorama actual, por lo que podemos afirmar que con la primera encuesta sobre FPI realizada en México cumplimos en parte con este primordial cometido. Sin embargo, tenemos que reconocer que los resultados pueden tener algunas limitantes, de las cuales vale la pena mencionar que de 500 neumólogos del país sólo respondieron 168 y de éstos la gran mayoría se sitúan en el centro del país. Asimismo, también es necesario mencionar que a diferencia de la encuesta europea, ${ }^{7}$ recién reportada, nuestra población de encuestados involucró a neumó- 


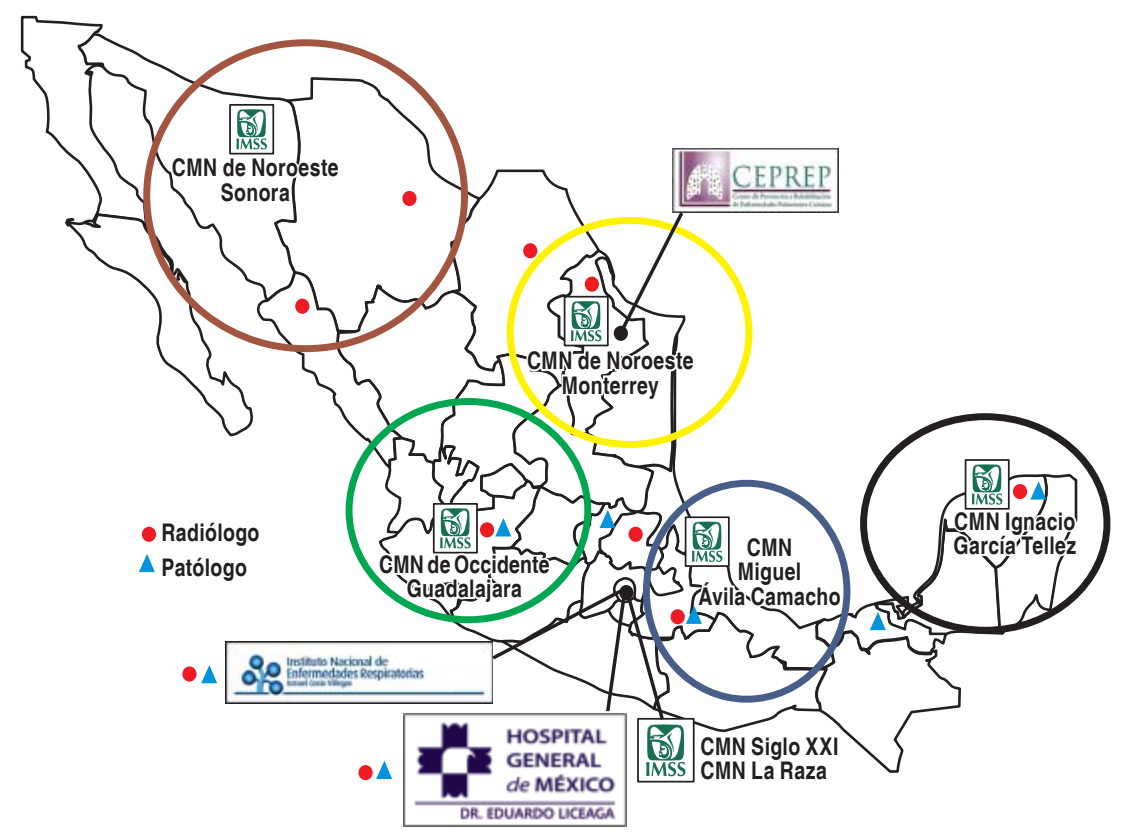

Fuente: Primera encuesta nacional de fibrosis pulmonar idiopática.

Figura 4.

Centros de referencia nacional para enfermedades intersticiales pulmonares.

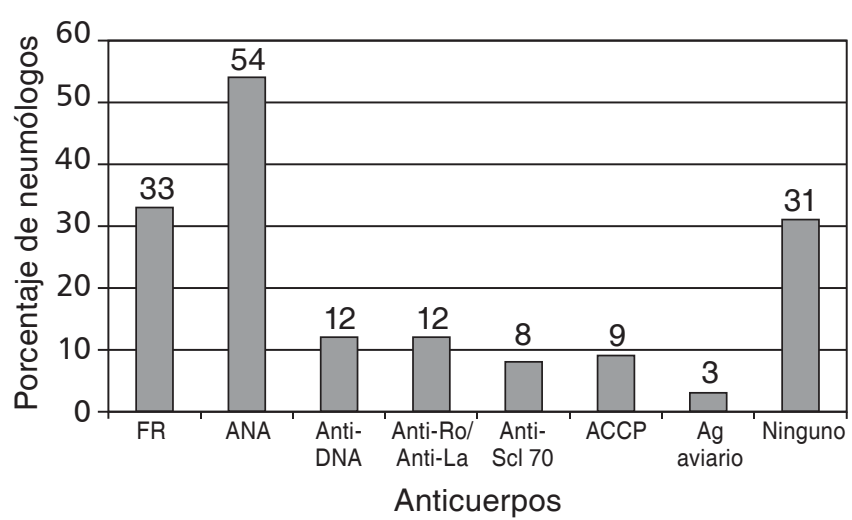

Fuente: Primera encuesta nacional de fibrosis pulmonar idiopática.

Figura 5. Anticuerpos solicitados por los neumólogos para descartar colagenopatías.

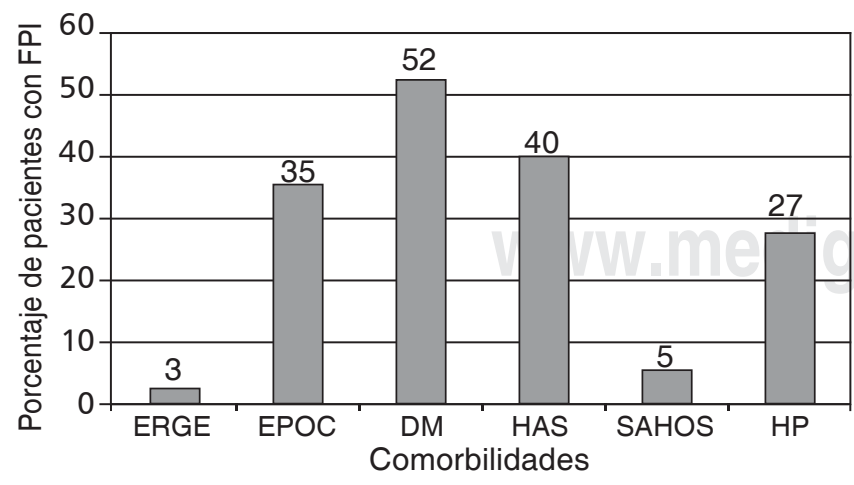

Fuente: Primera encuesta nacional de fibrosis pulmonar idiopática.

Figura 6. Comorbilidades en pacientes con FPI.

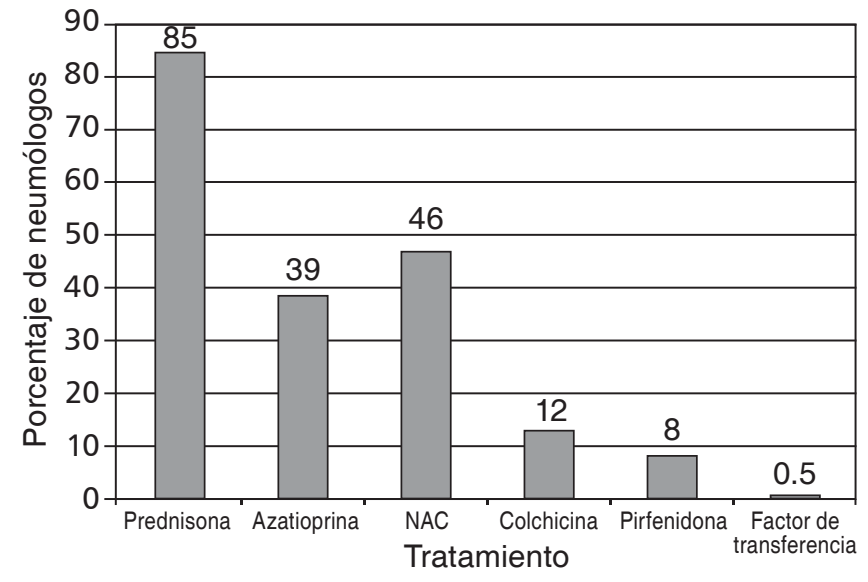

Fuente: Primera encuesta nacional de fibrosis pulmonar idiopática.

Figura 7. Terapia de inicio en FPI en México.

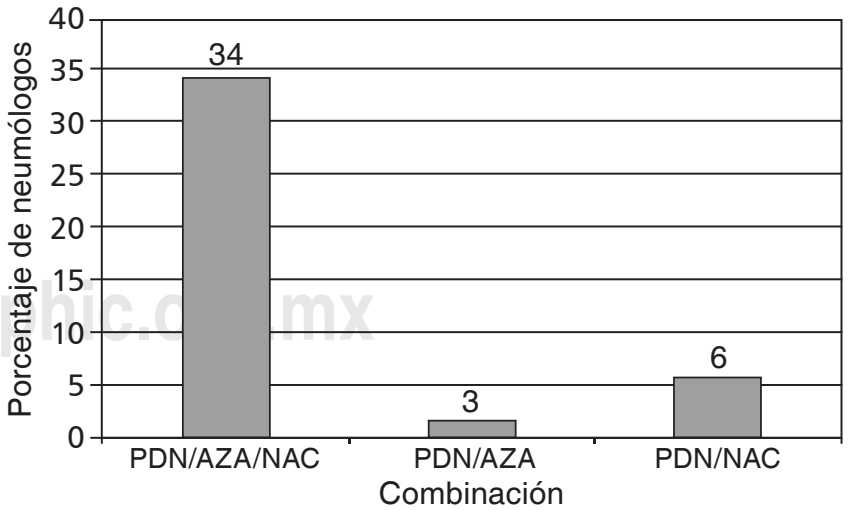

Fuente: Primera encuesta nacional de fibrosis pulmonar idiopática.

Figura 8. Combinaciones utilizadas como terapia de inicio en FPI en México. 
logos no necesariamente expertos en FPI, por lo que el panorama mostrado refleja la situación real sobre el abordaje de la FPI en México.

Con respecto a la frecuencia con la que los neumólogos de México diagnostican la enfermedad observamos que en promedio cada médico encuestado diagnostica 8 pacientes por año. Pero al observar este resultado por región del país podemos notar que incluso en estados próximos existen variaciones importantes entre sí, por ejemplo, en el Distrito Federal, la frecuencia de diagnóstico es de 6-10 pacientes por año, mientras que en el Estado de México y en el estado de Puebla la frecuencia aumenta hasta más de 15 pacientes por año. Situación que puede representar un sobrediagnóstico de FPI en estos estados o un subdiagnóstico en el Distrito Federal.

Un dato importante que reveló la encuesta es el hecho de que la gran mayoría de los neumólogos del país no envían pacientes a alguno de los centros de referencia para enfermedades intersticiales del pulmón, lo que resulta en mayor dificultad para el abordaje de la enfermedad e impide a los pacientes el ingreso a ensayos clínicos; al mismo tiempo, limita la formación de recursos humanos expertos en el tema.

Por otro lado, aun cuando la gran mayoría de los neumólogos disponen de TAC-AR, el abordaje diagnóstico difícilmente se realiza por un equipo multidisciplinario, ya que la cantidad de radiólogos y patólogos expertos en enfermedades intersticiales pulmonares son pocos, realidad que involucra no sólo a México, sino también a Latinoamérica tal y como lo mostró la encuesta desarrollada por ALAT en $2013,{ }^{8}$ así que es importante favorecer las altas especialidades en enfermedades intersticiales. Asimismo, hay que hacer notar que una tercera parte de los neumólogos encuestados no consideran necesario solicitar autoanticuerpos para descartar enfermedades autoinmunes como posibles causas de la fibrosis pulmonar, y son aún menos los que descartan enfermedades exposicionales como la neumonitis por hipersensibilidad; esto probablemente debido a que la disponibilidad de antígenos específicos es poco frecuente y ejemplo claro de ello es el antígeno aviario que sólo se realiza en el INERI. Sin embargo, aun con estas limitantes debe recordarse que el primer criterio para corroborar el diagnóstico es descartar otra enfermedad intersticial como causa de la fibrosis.

En cuanto al manejo del paciente con FPI pudimos observar que sigue siendo a base de corticoides, azatioprina y acetilcisteína, situación por demás preocupante, ya que pese a la alarma del estudio panther ${ }^{9}$ publicada en 2011, la terapéutica con el triple esquema sigue siendo frecuente. Esta problemática puede tener su origen en el hecho de que al momento de realizar la encuesta, aún no estaba disponible en México ningún medicamento especifico para FPI, ni ha salido alguna actualización de tratamiento por parte de las sociedades neumológicas, además, el estatus socioeconómico de la población mexicana no es el suficiente como para disponer de medicamentos especializados.

Finalmente podemos concluir que México no sólo necesita formar neumólogos expertos en enfermedades intersticiales, sino que también será fundamental incrementar la cantidad de radiólogos y patólogos expertos en el tema, así como formar una red adecuada de centros de referencia nacional que les permita a los pacientes un diagnóstico temprano y un manejo terapéutico adecuado; y para ello, jugará un papel importante la educación médica continua.

\section{Agradecimientos}

Al apoyo de Boehringer-Ingelheim México y Dr. Alberto Hegewis y Juan Ignacio Ortiz para la aplicación de la encuesta durante el congreso Nacional de Neumología y Cirugía de Tórax y el desarrollo de este trabajo.

\section{REFERENCIAS}

1. Raghu G, Collard HR, Egan JJ, et al; ATS/ERS/JRS/ ALAT Committee on Idiopathic Pulmonary Fibrosis. An official ATS/ERS/JRS/ALAT statement: idiopathic pulmonary fibrosis: evidence-based guidelines for diagnosis and management. Am J Respir Crit Care Med 2011;183(6):788-824. doi: 10.1164/rccm.2009-040GL.

2. Selman M, King TE, Pardo A; American Thoracic Society; European Respiratory Society; American College of Chest Physicians. Idiopathic pulmonary fibrosis: prevailing and evolving hypotheses about its pathogenesis and implications for therapy. Ann Intern Med 2001;134(2):136-151.

3. Ley B, Collard HR. Epidemiology of idiopathic pulmonary fibrosis. Clin Epidemiol 2013;5:483-492. doi: 10.2147/ CLEP.S54815.

4. Richeldi L, du Bois RM, Raghu G, et al; INPULSIS Trial Investigators. Efficacy and safety of nintedanib in idiopathic pulmonary fibrosis. N Engl J Med 2014; 370(22):2071-2082. doi: 10.1056/NEJMoa1402584.

5. King TE Jr, Bradford WZ, Castro-Bernardini S, et al; ASCEND Study Group. A phase 3 trial of pirfenidone in patients with idiopathic pulmonary fibrosis. N Engl J Med 2014;370(22):2083-2092. doi: 10.1056/NEJMoa1402582.

6. Collard HR, Moore BB, Flaherty KR, et al; Idiopathic Pulmonary Fibrosis Clinical Research Network Investigators. Acute exacerbations of idiopathic pulmonary fibrosis. Am J Respir Crit Care Med 2007;176(7):636-643.

7. Cottin V. Current approaches to the diagnosis and treatment of idiopathic pulmonary fibrosis in Europe: the AIR survey. Eur Respir Rev 2014;23(132): 225-230. doi: 10.1183/09059180.00001914. 
8. Curbelo P. Encuesta de EPID en Latinoamérica-ALAT 2013. Respirar 2013;5 (2):5-8.

9. National Heart, Lung and Blood Institute. Commonly used three-drug regime for idiopathic pulmonary fibrosis found harmful. Date last accessed: November 2, 2011. Date last updated: October 21, 2011. Available from: www.nih-gov/news/health/oct2011/nhlbi-21.htm. $\triangle$ Correspondencia:

Dra. Ivette Buendía-Roldán

Instituto Nacional de Enfermedades Respiratorias Ismael Cosío Villegas. Calzada de Tlalpan Núm. 4502, Colonia Sección XVI, 14080, México, D.F. Correo electrónico: ivettebu@yahoo.com.mx

Los autores declaran no tener conflicto de intereses. 